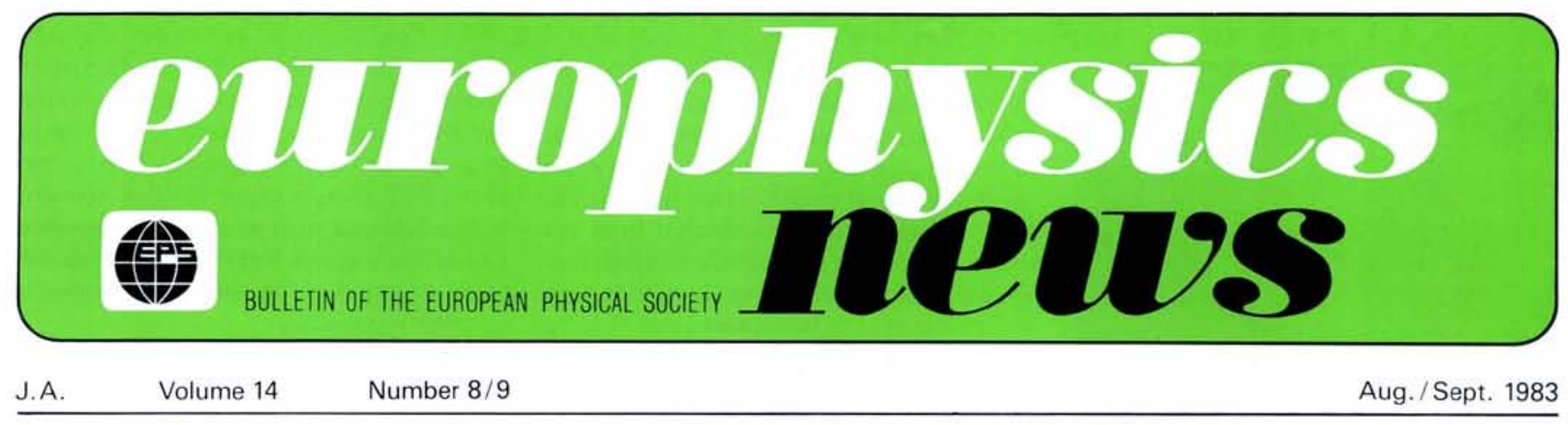

\title{
Current Problems in Macromolecular Physics
}

\author{
A. Keller, Bristol \\ (H.H. Wills Lab., University of Bristol)
}

and

\author{
H.H. Kausch, Lausanne \\ (Lab. de Polymères, Ecole Polytechnique Fédérale de Lausanne)
}

The characteristic properties of macromolecules are their length (up to tens of thousands of covalently bound atoms), their anisotropy and their mobility. The constraints imposed by the coupling of so many atoms, the importance of the configurational entropy and the relative weakness of the intermolecular attraction give rise to a wealth of interesting and specific physical phenomena.

It took exactly thirty years from the first conception of the notion of a macromolecule (by Staudinger in 1921) to the firm establishment of the physical chemistry of polymers given by Flory in 1951. During this time, much of the work centred around the behaviour of chain molecules in solution (viscosity, conformation), an elucida-

Fig. 1 - Electron micrograph of a typical single crystal of polyethylene crystallized from solu. tion. These crystals contain the chain in a folded conformation where the chain direction in the straight fold stems are perpendicular to the lamellar surfaces, the lamellar thickness thus defining the fold length (Kellet $A$. and $O^{\prime}$ Connor A., Disc. Faraday Soc. 25 (1958) 114).

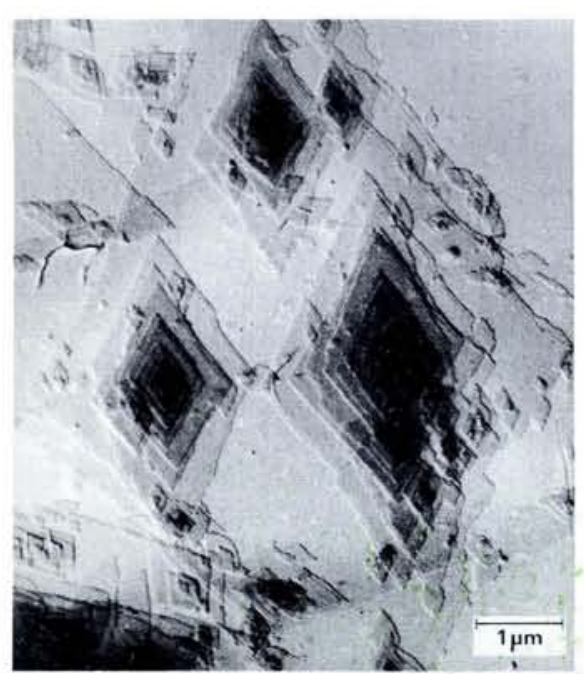

tion of their structure, morphology and state, and a comprehension and mathematical description of their macroscopic properties (the concept of the statistical theory of rubbery networks by W. Kuhn dates from 1934)

The present situation of macromolecular physics is characterized by the fact that the number and complexity of polymers available has increased considerably, that polymer blends (mixtures, copolymers, filled systems) have gained a wide-spread importance, and that new methods have opened completely new fields of investigation (just one example: neutron scattering techniques). In view of the limited space, the authors of this report would like to content themselves with sketching some of the current activities concerning solid polymers. In fact, the programme of the last EPS Conference on Macromolecular Physics in Vilafranca in 1982 (see refs. 1, 2) and of the up-coming conference (in Hamburg, 20-23 September 1983) offer an excellent frame of discussion.

The subject chosen by the Vilafranca Conference: "Polymer Crystals: Structure and Morphology" is one of the liveliest in polymer science and in fact in the physics of solid materials. As the title says, it concerns the structure and morphology of polymer crystals. First a few words about the subject matter.

\section{Crystallization}

It has long been known that polymer molecules consisting of identical repeat units can crystallize. The crystal lattice this gives rise to and the position of the atoms within it have long been pursued by traditional methods of X-ray crystallography. This traditional "crystal structure analysis" has not, however, been directly concerned with issues such as how the molecule along its full length is to be visualized in relation to the crystal lattice. The impor- tance of the latter issue has become highlighted over the last 25 years by the discovery that crystalline high polymers possess a striking and varied morphology on the submicroscopic and microscopic level which is closely linked to the arrangement and behaviour of long chains on the molecular level, that is with the issue of "crystal structure" in the broader sense. It is this wider subject area, rather than the more narrowly defined traditional definition of crystal structure which was the subject of the Conference.

A concrete example of this interconnection between molecular structure and morphology, which was also one of the central subjects of the Conference, is the lamellar nature of the basic crystal entities, containing the polymer chains in a folded conformation where the thickness of the lamellae (in the range of about $10-50 \mathrm{~nm}$ ) corresponds to the fold length. This provides a salient example, totally unique in crystallography or in the structural science of solid matter, where a morphological feature (crystal thickness) and a molecular feature (chain folding) is inextricably linked. The

\begin{tabular}{|c|c|}
\hline Contents & \\
\hline $\begin{array}{l}\text { Current Problems in } \\
\text { Macromolecular Physics }\end{array}$ & 1 \\
\hline $\begin{array}{l}\text { Synthesis of Nuclei at the } \\
\text { Limits of Nuclear Stability }\end{array}$ & 4 \\
\hline University-Industry Interactions & 8 \\
\hline New EPS Members & 8 \\
\hline Helping Developing Countries & 9 \\
\hline International Physics & 9 \\
\hline CMD Elections & 12 \\
\hline
\end{tabular}




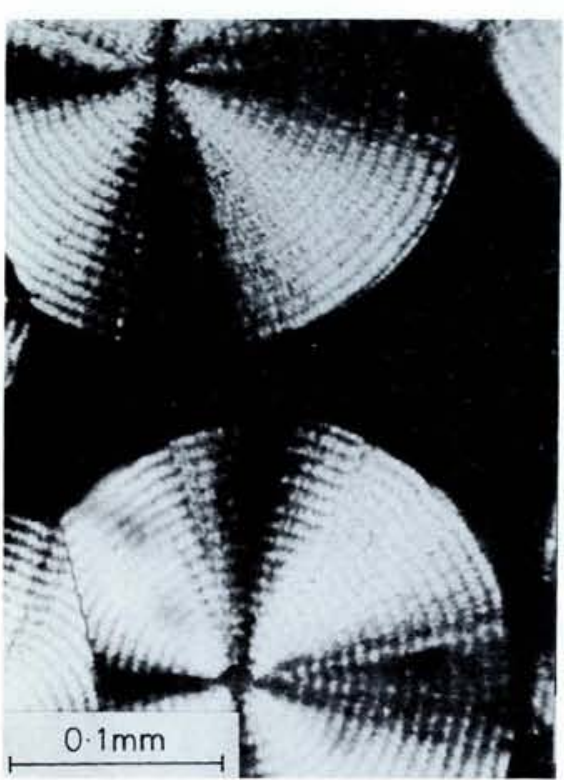

Fig. 2 - Photomicrograph taken between cross ed polaroids capturing one stage of the crystallization of polyethylene. The circular (spherical in three dimensions) birefringent objects are the spherulites representing the crystal entities growing within the amorphous melt. They are not individual crystals but assemblies of crystal lamellae arranged in a spherically symmetrical manner, where the lamellae are growing radially outwards from a common centre with an inbuilt periodic twist (from Chivers R.A., Barham P.J., Martinez-Salazar J. and Keller A., J. Polymer Sci. Physics Ed. 20 (1982) 1717).

topicality of such and related issues arises from the fact that we do not know why macromolecules, even the simplest kind (e.g. polyethylene), behave as observed (e.g. fold) when forming crystals, neither what is exactly the nature of the full chain trajectory in molecular or atomic detail. Beyond that is the question of how can long, presumably highly entangled chains form such regular organizations as are in fact observed, while still retaining appreciable amounts of disorder (amorphous content) which imparts the familiar "plastic like properties" to plastic objects such as are known in everyday life. Such issues together with many others are subjects of acute controversy.

One source of these controversies can be traced to the fact that no single enquiry on its own can produce a unique answer to the various questions. Consequently, a combination of different approaches and experimental techniques is required, the importance of each being weighted differently by those holding opposing views. It is this unusually varied and kaleidoscopic nature of the problems, with all its important implications, which made the Vilafranca Conference, superbly organized by Professor Baltà-Calleja, so topical, lively and hopefully productive.

As indicated above, lamellar crystals and chain folding is the central theme. For any kinetic considerations it is important to know the fold length at the initial stage of deposition as opposed to its altered (refolded) version, the quantity usually observed, at least in crystallization from melts. Recent results by one of us (AK) show how the initial fold length can be extracted from the experimental data on altered (refolded) material providing both a direct base for future theoretical efforts to explain chain folding and also for the understanding of the refolding process itself.

Much progress came from the application of neutron scattering techniques ${ }^{3}$ ). A major issue still is the nature of the chain trajectory and the question to what extent the chains fold back on themselves in an adjacently or non-adjacently reentrant manner. Results from scattering experiments recently accomplished on polyethylene and supercooled polystyrene seem to form a bridge between some of the conflicting views on the whole subject of fold reentry and global conformation of a given chain within the crystal. Wittmann and Lotz (Strasbourg) have developed a new, highly original technique, namely decoration of polymer crystal surfaces by vapourphase deposited low molecular weight polyethylene crystals with striking demonstration of an epitaxial relation between the fold surface of the subtrate crystal and the deposited overgrowth. This was possibly the most original finding reported at the Conference with direct relevance to the central issues concerning the regularity of the fold surface.

The promotion of crystal nucleation by foreign additives, the effect of chemical or conformational chain defects and of chain length differences on crystal perfection claim wide interest. The latest developments of Hosemann's theory on paracrystals with special emphasis on the universal relation between paracrystalline order and mosaic crystal block size and the potential connection between paracrystallinity and dislocations should be mentioned. An interesting new aspect constitutes the role of reptation in the crystallization of polyethylene, the main thrust of this message being that, taking reptation into account, the intrinsic mobility of the chains suffices to account for the formation of chain folded crystals and for their observed, in some cases surprisingly high growth rate (Hoffmann).

\section{Orientation and Mobility}

Finally, the important problem area of polymer orientation should be mentioned. It is characteristic of chain molecules that their equilibrium conformation is that of a statistical coil, the diameter of which is only a fraction of the extended length of the molecule. By "drawing" such a sample to more than 20 times its original length, fibrillar structures are obtained with extremely interesting high moduli and high strength values - comparable even to those of steel. A new route for preparing ultra high modulus fibres is via formation and subsequent drawing of gels where special emphasis must be given to the role of entanglements. The booklet of extended abstracts ${ }^{1}$ ) gives a more detailed account of the subjects treated at this Conference and of the authors and (European) laboratories engaged in the very active research on polymer crystals.

The above discussion of molecular dynamics, order and orientation will be continued at the Hamburg meeting; it will be extended to amorphous materials, and special emphasis will be placed on new developments in methods of characterization of polymers in the solid state.

As indicated in the introduction, molecular mobility is one of the most important parameters. The term "one parameter" is a great understatement, since one has to consider the mobility on every structural level: the motion of atoms, atom groups, chain segments and of entire chains. A relatively new and powerful method to analyse the local chain structure and the transitions between different conformations by rotation about single bonds is the ${ }^{13} \mathrm{C}-\mathrm{NMR}$ technique $\left.{ }^{4}\right)$. The information obtained this way is needed to understand the dielectric and mechanical relaxation behaviour of polymers, enabling the microstructure of copolymers to be described.

A second subject of great topicality (and for which Hamburg offers particularly excellent facilities) is the use of synchrotron radiation $\left.{ }^{4}\right)$. The interest of polymer science in such a powerful X-ray source stems from the fact that rapid transient phenomena like crystal orientation or break-up can hardly be studied otherwise. Due to the pioneer work of Stein (Amherst) "conventional" small angle $X$-ray scattering techniques (SAXS) have been applied, however, to analyse the cyclic deformation behaviour of semi-crystalline polymers. Widespread use is made of SAXS to study the (quasi-static) colloidal structure of polymers such as long periods in lamellar systems, defect sizes in irreversibly deformed (voided) polymers, or the fibril diameters in crazes.

The preceding discussion of crystal physics, molecular mobility and colloidal structure has made abundantly clear that the question of how polymer solids are organized is of interest to all domains of polymer physics. This argument is valid even if the electrical and optical properties or the mechanical properties of amorphous materials are concerned. The authors wish to conclude this article with a discussion of some recent research of their own special interest on the "structure" and deformation of amorphous polymers.

\section{Amorphous Polymers}

An isotropic thermoplastic amorphous polymer can be considered as a physical network of statistically coiled, entangled molecules. The normal response of such a material to increasing loads will be an 


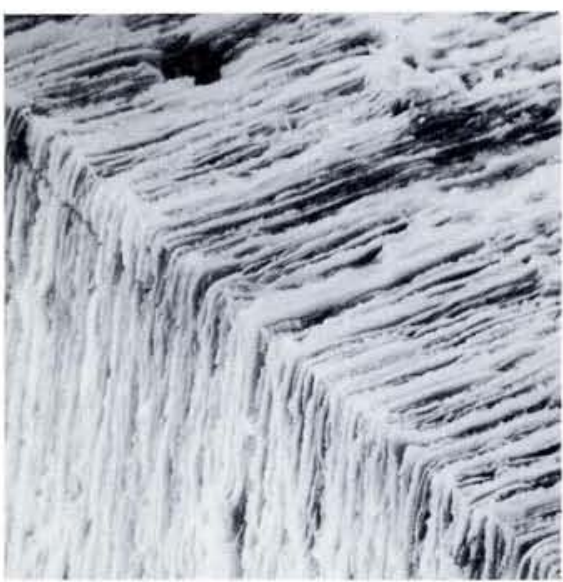

Fig. 3 - Intrinsic crazes in polycarbonate (Kausch H.H. and Dettenmaier, Ref. 5).

anelastic widening of the "lattice", bond rotation in the main chain leading to a change of conformation and creep, segment rotation and craze initiation (where appropriate), and a brittle (fracture) or ductile instability (segment slip and yielding followed by large scale chain orientation and either strain hardening or flow). In the presence of a superstructure, these phenomena will preferentially develop at the intergranular boundaries. Whereas the elastic and anelastic properties are very often determined by the interaction of the individual segments, there is no doubt that the ultimate properties depend on the structure of the network as a whole.

The characteristic parameters of an amorphous network are evidently packing density, orientation distribution and correlation of chain segments, distribution of entanglement and crosslinking points and density of chain ends. These parameters fluctuate in space and time. Since each of them influences the mechanical response of a material, there will, in principle, be a fluctuation of the local strength of the network. The recent studies of crack healing and chain diffusion at interfaces by one of us (HHK, see ref. 4) have indicated, however, that entanglements between molecules stabilize the stress transfer between segments and prevent their rapid separation. In those plastics used for load bearing applications, there will generally be more entanglements per molecule than needed to stabilize rapid stress transfer. Small local fluctuations of entanglement density, therefore, seem to be of no consequence. If, however, the number of entanglements per molecule is decreased then there will be volume elements with uncoupled molecules which constitute defect sites. A decrease of entanglement concentration can occur through chain scission, flow or long-time loading or through fatigue loading.

A final word may be said with respect to a phenomenon characteristic of loaded glassy polymers: the formation of crazes, small voided zones opening up normal to the direction of the largest principal stress component and spanned by fibrillar strands of oriented molecular coils ${ }^{5}$ ). The conventional or extrinsic crazes are initiated in the neighbourhood of flaws or dust particles and/or by traces of crazing agents preferentially at the specimen surfaces.

\section{Craze Formation}

Craze formation has been studied for over thirty years now and by a large number of researchers. Their accumulated efforts have been prerequisite to the most recent progress obtained by the use of two specific methods (see ref. 5 for extended articles on this subject). These are first the electron beam imaging technique developed in Ithaca (by E.J. Kramer and collaborators) which has proved to be an extremely powerful tool in determining the micromechanics, i.e. the shape and state of stress of a single craze in a thin film. Thus, considerable information has been gained with respect to craze nucleation, microstructure and growth. One of the most important craze parameters, the local fibril extension ratio has become amenable to study. Kramer has investigated the influence of molecular entanglements for a wide variety of glassy polymers with chains of different entanglement molecular weight. He concludes that under special circumstances chain scission can have a significant role in the break down of craze fibrils.

The second method which recently provided considerable insight into the role of crazes in deformation and fracture of amorphous polymers is the optical interference measurement of crazes (preceding a crack). Since the pioneer work of Kambour, this method has been widely used to determine characteristic craze dimensions and critical displacements. W. Döll (Freiburg/Breisgau) and I.M. Ward (Leeds) thus relate molecular parameters (chain length and mobility) with macroscopic and fracture mechanics parameters (stress intensity factor, plastic zone sizes, fracture surface morphology, fracture energy).

As opposed to extrinsic crazing, the formation of intrinsic crazes (crazes II), as recently observed by Dettenmaier (Lausanne
$4,5)$, clearly constitutes an instability which depends on the network properties and is not restricted to the sample surface. It has been demonstrated that crazes II are initiated in originally unoriented PC at an extension ratio of about $\lambda^{\prime \prime}=2.1$. This value agrees rather well with the maximum extensibility of chains between entanglement points, $\lambda^{\text {nat }}$. Therefore, it must be assumed that disentanglement of chains either by chain slippage or by chain rupture plays an important role in craze II formation and growth.

\section{Conclusion}

In summary one may say that the chainlike nature of macromolecules is giving rise to a large variety of structures embracing a wide range of structure hierarchies from the molecular to the macroscopic level with numerous variants within each. The exploration of these structures and the understanding of how and why they arise is the subject of fundamental research in polymer physics stemming from basic scientific curiosity. From the point of view of application the above mentioned wealth of structural organizations is not only a source of complexity but also offers a virtually unlimited store of potential to control and design physical behaviour for specific purposes, a potential which is far from being fully exploited at present. Some of the principal areas of research in the field of the physics of polymers aim at furthering our knowledge in this direction. The knowledge thus gained increases our capability to exploit the virtually limitless potential this offers for applications.

\section{REFERENCES}

1. "Polymer Crystals: Structure and Morphology", Europhysics Conference Abstracts 6G (1982).

2. Baltà-Calleja F.J., Europhysics News 13 (1982) $12,10$.

3. Kausch H.H., Europhysics News 9 (1978) 9,8 . 4. "New Developments in the Characterization of Polymers in the Solid State", Europhysics Conference Abstracts 7G (1983).

5. "Crazing in Polymers", ed. H.H. Kausch, Advances in Polymer Science Vol. 52/53 (SpringerVerlag, Berlin-Heidelberg) 1983.

\section{UNIVERSITY OF GENEVA}

The Department of Nuclear and Particle Physics has an opening for a position of

\section{RESEARCH ASSOCIATE}

(maître-assistant) in high energy physics. This is a non permanent position limited to a maximum of 6 years. The candidate must have a PhD (or equivalent title).

Applications should be sent to the Director of Département de Physique nucléaire et corpusculaire, 32, bd d'Yvoy, $\mathrm{CH}-1211$ Geneva 4 